\section{Detailed tabulation of atomic form factors, photoelectric absorption and scattering cross section, and mass attenuation coefficients in the vicinity of absorption edges in the soft $X$-ray $(Z=30-36, Z=60-89$, $E=0.1-10 \mathrm{keV}$ ) - addressing convergence issues of earlier work}

\section{T. Chantler}

School of Physics, University of Melbourne, Victoria 3010, Australia.E-mail: chantler@physics.unimelb.edu.au

A new tabulation of atomic form factors is discussed briefly, extending the validity of the isolated atom approximation and serving as a baseline for near-edge solid-state and XAFS investigations. This is detailed by Chantler [J. Phys. Chem. Ref. Data, (2000), 29, 597-1048] and is the latest component of the FFAST tabulation of NIST.

Keywords: anomalous dispersion; atomic photoeffect; attenuation coefficients; cross sections; form factors; photons; scattering factors; X-rays.

Reliable knowledge of the complex X-ray form factor $\left[\operatorname{Re}(f)\right.$ and $\left.f^{\prime \prime}\right]$ and the photoelectric attenuation coefficient $\left(\sigma_{\mathrm{PE}}\right)$ is required for crystallography, medical diagnosis, radiation safety and XAFS studies. Discrepancies of $200 \%$ between the currently used theoretical approaches of Scofield (1973), Saloman \& Hubbell (1986), Henke, Gullikson \& Davis (1993), Saloman, Hubbell \& Scofield (1988) and Chantler (1995) exist for numerous elements for X-ray energies from 1 to $3 \mathrm{keV}$. This level of inconsistency may be surprising to some users who have conventionally viewed $\log -\log$ plots covering decades in energy and attenuation coefficient, but these discrepancies have been present in the literature for decades.

Chantler (2000) identifies key discrepancies due to the smoothing of edge structure, the use of non-relativistic wave functions, and the lack of appropriate convergence of wave functions. He addresses these discrepancies and finds that significant corrections are required for most comprehensive (i.e. all-Z) tabulations. The paper finds that the earlier work of Chantler (1995) needs improvement in the nearedge region for soft X-ray energies, and derives new theoretical results of substantially higher accuracy in near-edge soft X-ray regions. The grid size and spacing of the reported tabulation is given with synchrotron users in mind, where fine grids near edges are necessary and continuous energy scans are possible. All energies above $0.1 \mathrm{keV}$ and all elements to $Z=92$ were investigated in this computation, and only the listed energies and elements were found to need re-evaluation for this tabulation.

A detailed review of form factors, attenuation coefficients, their uses and a range of current tabulations is given. Estimates for the expected accuracy of the pair of publications [Chantler (1995) and Chantler (2000)] is given across the full range of $Z$ and energy, including near-edge limitations of wavefunction convergence and near-edge structure itself. Limitations of the current approach are also illustrated. An obvious point, for XAFS and MAD users, is the absence of near-edge structure, and perhaps a deviation of the edge onset from a particular solid-state system. This is an advantage, which serves to separate the oscillatory near-edge structure and discrete lines or near-edge zeroes from a reference baseline for the given element.

A summary is given of typical applications and how to use the tables, including a short discussion on sources of theoretical correction or imprecision. Six tables are provided, the last giving the coarse but standard Grodstein grid for the elements involved, which is convenient for comparison with earlier and sparser works. In all, 79 works are cited, with 89 figures illustrating detailed comparisons.

The energy range covered is $0.1-10 \mathrm{keV}$. The associated figures and tabulation demonstrate the current comparison with alternate theory and with available experimental data. In general, experimental data are not sufficiently accurate to establish the errors and inadequacies of theory at this level. However, the best experimental data and the observed experimental structure as a function of energy are strong indicators of the validity of the current approach. New developments in experimental measurement hold great promise in making critical comparisons with theory in the near future. This work forms the latest component of the FFAST tabulation of NIST.

\section{References}

Chantler, C. T. (1995). J. Phys. Chem. Ref. Data, 24, 71-643.

Chantler, C. T. (2000). J. Phys. Chem. Ref. Data, 29, 597-1048.

Henke, B. L., Gullikson, E. C. \& Davis, J. C. (1993). Atom. Data Nucl. Data Tables, 54, 181-342.

Saloman, E. B. \& Hubbell, J. H. (1986). Natl. Bur. Stand. Intern. Rep. pp. 863431.

Saloman, E. B., Hubbell, J. H. \& Scofield, J. H. (1988). Atom. Data Nucl. Data Tables, 38 1-197.

Scofield, J. H. (1973). LLNL Report UCRI-51326. Lawrence Livermore National Laboratory, CA 94550-9234, USA. 